\title{
Bcl-2 inhibits the caspase-dependent apoptosis induced by SARS-CoV without affecting virus replication kinetics
}

\author{
Brief Report \\ L. Bordi ${ }^{1}$, C. Castilletti ${ }^{1}$, L. Falasca ${ }^{2}$, F. Ciccosanti ${ }^{2}$, S. Calcaterra ${ }^{1}$, \\ G. Rozera ${ }^{1}$, A. Di Caro ${ }^{1}$, S. Zaniratti ${ }^{1}$, A. Rinaldi ${ }^{2}$, G. Ippolito ${ }^{3}$, \\ M. Piacentini ${ }^{2,4}$, and M. R. Capobianchi ${ }^{1}$ \\ ${ }^{1}$ Laboratory of Virology, National Institute for Infectious Diseases \\ INMI "L. Spallanzani”, Rome, Italy \\ ${ }^{2}$ Laboratory of Cell Biology and Electron Microscopy, National Institute \\ for Infectious Diseases INMI "L. Spallanzani”, Rome, Italy \\ ${ }^{3}$ Department of Epidemiology, National Institute for Infectious \\ Diseases INMI "L. Spallanzani”, Rome, Italy \\ ${ }^{4}$ Department of Biology, University of Rome "Tor Vergata", Rome, Italy.
}

Received November 1, 2004; accepted August 2, 2005

Published online September 12, 2005 (C) Springer-Verlag 2005

Summary. Vero cells transfected with either neo- or $b c l$-2-plasmid were infected with SARS-CoV at a high multiplicity of infection. Apoptosis appeared after the onset of CPE and completion of virus replication, and could be prevented by Bcl-2 expression. Apoptosis is likely mediated by the mitochondrial pathway, as demonstrated by its inhibition using $\mathrm{Bcl}-2$, and by the activation of the caspase cascade, resulting in PARP cleavage. Prevention of apoptosis did not affect susceptibility to infection, kinetics and extent of viral replication and release, thus implying that apoptosis is not involved in facilitating release and/or dissemination of SARS-CoV in Vero cells.

$$
*
$$

Apoptosis is an important antivirus defence mechanism of host cells, and viruses have evolved strategies to counteract and regulate apoptosis to maximize the production of virus progeny, allowing viral spread to neighbouring cells [11]. Several cytopathic viruses are able to affect the apoptotic process either through induction or inhibition mechanisms, and it is generally believed that apoptosis, besides necrosis, can mediate the cytopathic effect (CPE) induced by some viruses [8]. In recent years, many viruses have been found to induce apoptosis during 
replication [11]. The molecular pathways used by viruses to induce apoptosis are still poorly understood. For some viruses, a viral protein has been identified as an apoptosis-inducing factor $[23,25]$. Alternatively, genes of many DNA viruses have evolved to encode proteins which suppress or delay apoptosis, thus facilitating the production of large amounts of progeny virus. For example, the poxvirus $\mathrm{crmA}$ gene product and baculovirus p35 prevent induction of apoptosis through the inhibition of cell death central effector machinery [5, 26]. Also, over-expression of the anti-apoptotic gene $b c l-2$ prevents either the Sindbis virus- or Semliki Forest virus-induced apoptosis, leading to the establishment of a persistent infection with increased virus production [13,14,22]. The role of apoptosis in the pathogenesis of coronavirus infections is not completely understood, although many data, obtained for both animal and human coronaviruses, indicate that apoptosis is associated with virus replication[1, 2, 4, 6, 9, 10, 12, 15, 19].

The recently discovered coronavirus, responsible for the human severe acute respiratory syndrome (SARS-CoV) [3, 20], grows faster than other known human coronaviruses and induces dramatic ultrastructural changes in host cells [17, 21], but the mechanisms that control the death of infected cells are still poorly understood. Yan et al. [27] have shown that the CPE induced by SARS-CoV in Vero E6 cells is mainly dependent on the activation of apoptosis. Recently, the viral accessory protein $7 \mathrm{a}$ has been found to be able to induce apoptosis via caspasedependent pathway [24]. However, it is still not clear whether apoptosis is required for release of virions by infected cells, and whether preventing apoptotic cell death could modify the efficiency and/or kinetics of virus replication.

In this study, we investigated whether inhibition of cell apoptosis would affect CPE appearance, as well as kinetics and extent of virus progeny production and/or release in Vero cells infected with SARS-CoV. To this aim, a Vero cell line that expresses Bcl-2- was obtained by transfecting Vero cells with the expression vector pEFbcl-2 (carring the human $b c l-2$ gene, kindly provided by Susan Cory, University of Melbourne, Australia) using the Lipofectin reagent (Gibco, Invitrogen Corporation, Carlsbad, Ca, USA); bulk transfectants were selected in the presence of $1 \mathrm{mg} / \mathrm{ml} \mathrm{G} 418$ (Gibco, Invitrogen Corporation, Carlsbad, Ca, USA). The control vector pEFneo was used to establish a control cell line. Expression of the transfected gene was stable after passaging these cells several times. Before study initiation, cells were grown for three weeks in the presence of G418, and Bcl-2 expression was confirmed by immunohistochemical staining and western blot analysis.

The Tor2 isolate of SARS-CoV (kindly provided by National Microbiology Laboratory, Public Health Agency of Canada) was used. To synchronize virus replication in the majority of cells, a high multiplicity of infection (MOI: tissue culture infectious doses $\left(\mathrm{TCID}_{50}\right) / \mathrm{cell}$ ) of 10 was used, simulating conditions of a single replication cycle. Mock-infected neo- and bcl-2-Vero cells were used as control cultures. In a parallel series of experiments, infection was carried out in neo-Vero cells that were treated with $20 \mu \mathrm{M}$ of the pan-caspase inhibitor Z-VAD-FMK (Sigma-Aldrich, St. Louis, MO, USA) after the absorption phase. 
Virus titration was performed by limiting dilution assay, using 10-fold serial dilutions. Both supernatants and cells lysates, obtained after 3 cycles of freezethawing followed by centrifugation, were used. CPE was detected after 5 days of infection, and titers were calculated according to the Reed and Muench formula [7].

Viral nucleic acids were extracted from either supernatants or cell lysates, using the NucliSens Isolation Reagents (NucliSensTM, Biomérieux, Hazelwood, MI, USA). Viral RNA was measured by real-time PCR, using the RealArt HPACoronavirus LightCycler RT PCR Reagents Assay (Artus GmbH, Hamburg, Germany) targeting the Pol gene, on the LightCycler instrument (Roche Diagnostics, Mannheim, Germany). Viral RNA concentration was calculated from a standard curve based on four external positive controls included in the kit. The kit included also a second heterologous amplification target as an internal control, based on a synthetic RNA oligonucleotide, also used for other Artus real time based kits [16], whose sequence is not publicly available.

For western blot analysis, cells were homogenized with lysis buffer $(50 \mathrm{mM}$ Tris-HCl [pH 8] containing $120 \mathrm{mM} \mathrm{NaCl}, 0.2 \mathrm{mM}$ EDTA, $1 \%$ NONIDET/ IGEPAL and protease inhibitor cocktail). Forty $\mu \mathrm{g}$ of protein extracts were run on $10 \%$ SDS-PAGE, in $25 \mathrm{mM}$ Tris, $192 \mathrm{mM}$ Glycine and $0.1 \%$ SDS, and electroblotted onto nitrocellulose membrane overnight at $4{ }^{\circ} \mathrm{C}$. Bcl-2, poly-(ADP-ribose)polymerase (PARP) and caspase- 8 bands were detected using rabbit polyclonal antisera specific for Bcl-2 (Abcam, Cambrige, UK) and PARP (Cell Signalling, Beverly, MA, USA), or with mouse monoclonal antibody (MAb) for caspase-8 (Cell Signalling). Tubulin expression was evaluated as an internal control, using mouse monoclonal antiserum specific for $\alpha$-tubulin (Sigma-Aldrich, St. Louis, MO, USA). A secondary horseradish peroxidase-conjugated goat anti-mouse or anti-rabbit antibody (Bio-Rad Laboratories, Hercules, CA, USA) was used as appropriate to bind primary antibodies. The signal was developed using the ECL chemiluminescence detection system (Amersham Biosciences, Uppsala, Sweden).

Cells were fixed in $4 \%$ paraformaldehyde for $20 \mathrm{~min}$. at room temperature and permeabilized with $0.1 \%$ Triton-X-100 in PBS for $20 \mathrm{~min}$, then washed with PBS. Endogenous peroxidase activity was inhibited using $3 \% \mathrm{H}_{2} \mathrm{O}_{2}$, and non-specific binding was blocked by incubating the cells with 5\% BSA in PBS for 20 min. Immunocytochemical staining was performed with rabbit antiserum specific for Bcl-2 (Abcam) and biotinylated goat anti-rabbit IgG. The immunoreaction product was detected using 3,3'-diaminobenzidine as chromogen substrate (SigmaAldrich) and $0.01 \% \mathrm{H}_{2} \mathrm{O}_{2}$. The cells were counterstained in Mayer's acid hemalum.

The detection of hypodiploid cells was based on propidium iodide staining techniques. Briefly, cells were detached by trypsinization and centrifuged; pellets were washed and fixed with $4 \%$ paraformaldehyde. After further washing, pellets were incubated with $100 \mu \mathrm{g} / \mathrm{ml}$ RNAsin (Promega, Madison, WI) for $20 \mathrm{~min}$. at room temperature and stained with propidium iodide $(50 \mu \mathrm{g} / \mathrm{ml})$ for $20 \mathrm{~min}$. Analysis was performed by flow cytometry using a FACScan Flow Cytometer (Becton-Dickinson, Franklin Lakes, NJ) with detection at 565 and $605 \mathrm{~nm}$ [18]. 

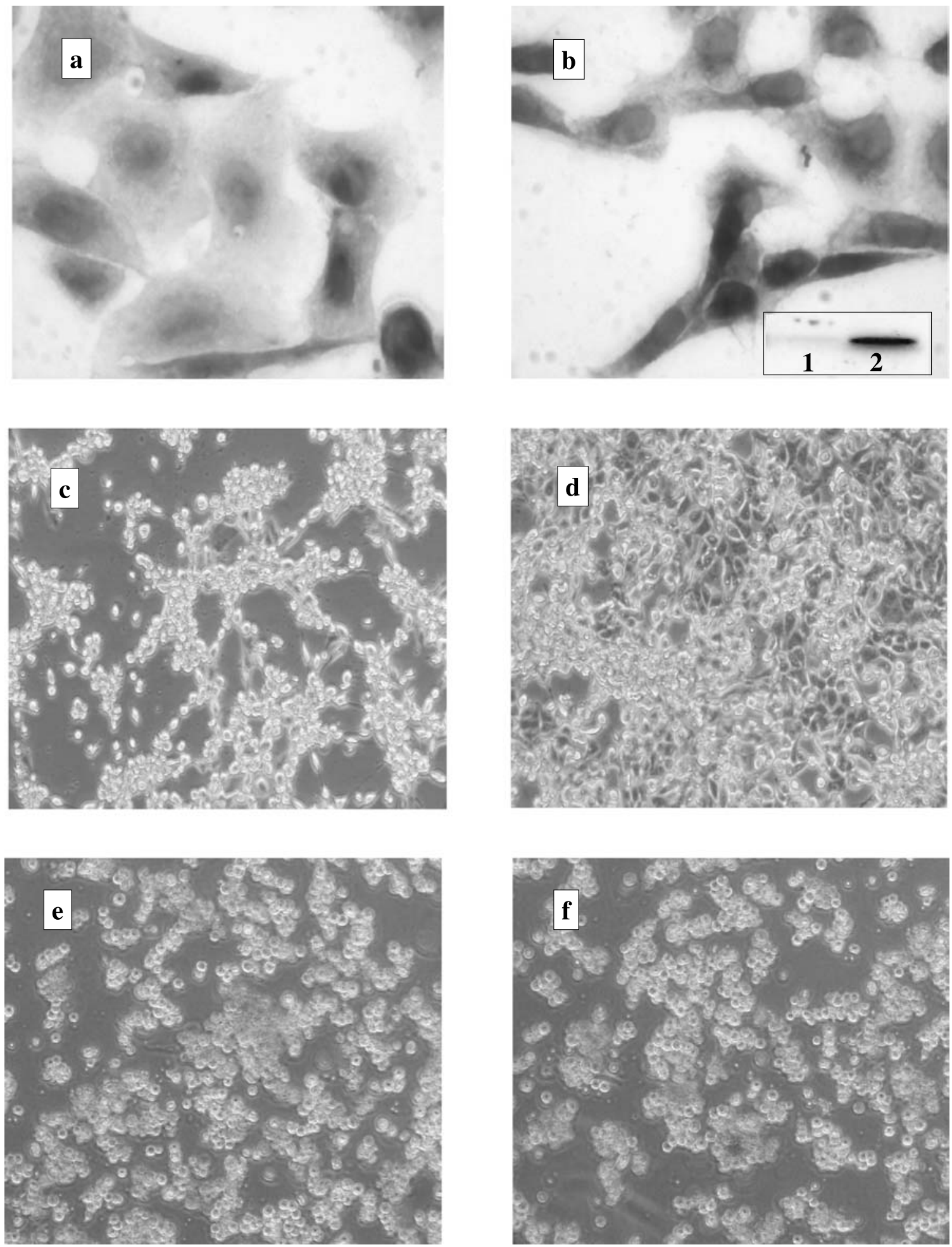

Fig. 1. Bcl-2 expression and SARS-CoV-induced CPE in transfected Vero cells. a, b) Immunochemical staining of Bcl-2 protein in Vero cells transfected with either control neo(a) or bcl-2-plasmid (b). Insert: western blot analysis of Bcl-2 expression in neo-Vero cells (1) and $b c l$-2-Vero cells (2). c-f) CPE appearance at 24 h.p.i. and 48 h.p.i. in neo- (c and e) and in $b c l$-2-Vero cells (d and $\mathbf{f})$. One representative experiment is shown 
Apoptosis was detected using the "In situ cell death detection kit" (Roche Diagnostics), based on the TUNEL (TdT-mediate d-UTP-biotin Nick End Labeling) assay, specific for labelling of cells with DNA breaks. Analysis was performed by flow cytometry at $517 \mathrm{~nm}$. For each sample, 10,000 events were acquired.

Each experimental point was run in duplicate, and representative results of at least three independent experiments are shown, unless otherwise specified. The statistical significance of the observed differences was evaluated by Student's t-test.

Our results confirm that pEFbcl-2-transfected Vero ( $b c l$-2-Vero), and not pEFneo-transfected Vero (neo-Vero) cells, express Bcl-2 (Fig. 1a and b). Under single replication cycle conditions, neo-Vero cells started to show CPE from 20 h.p.i., and at 24 h.p.i. almost the entire monolayer was affected (Fig. 1c), progressing toward complete shrinkage and rounding of the cells at $48 \mathrm{~h} . \mathrm{p} . \mathrm{i}$. In bcl-2-Vero cells CPE onset was delayed, with only part of the monolayer affected at 24 h.p.i. (Fig. 1d); however, from 48 h.p.i. onward, the extent of CPE was similar to that observed in neo-Vero cells (Fig. 1e and f).

The number of hypodiploid cells in neo-Vero cultures started to increase at 48 h.p.i., i.e. after CPE became evident ( 20 h.p.i.). The proportion of hypodiploid cells was lower in Bcl-2-expressing cells, remaining in the range of uninfected cells throughout entire observation period (Fig. 2a). The reduction was highly significant at 72 h.p.i. (34.4\% of hypodiploid cells in neo-Vero cells vs $8.7 \%$ in $b c l$-2-Vero cells; $\mathrm{p}=0.008$ ). Consistently, the frequency of TUNEL-positive cells was reduced to background levels in $b c l-2$-Vero cells $(25 \%$ in neo-Vero cells vs $9 \%$ in $b c l$-2-Vero cells at 48 h.p.i.) (Fig. 2b). The exposure of SARS-CoV infected cells to Z-VAD-FMK completely prevented apoptosis (not shown). Cleavage products of both PARP, a well known caspase- 3 substrate, and procaspase- 8 , were detected in SARS-CoV infected neo-Vero cells, and were partially prevented in bcl-2-Vero cells at 72 h.p.i. (Fig. 2c). These findings support the hypothesis that apoptosis is triggered by a caspase-dependent mechanism in our system.

Figure 3 shows the time course of infectious virus progeny production, either released in the supernatant or remaining cell-associated, in neo- and $b c l-2$-Vero cells cultures. During the logarithmic phase of viral replication (with a peak at 16 h.p.i. that was sustained to 24 h.p.i.), cell-associated infectivity was higher than that released in the supernatants in both culture systems. At 48 h.p.i., the proportion of cell-associated and released virus was similar, and at 72 h.p.i., the proportion was reversed. Interestingly, the time course and the extent of viral progeny production, as well as the proportion of cell-associated vs shed virus, was super-imposable for $b c l$-2- and neo-Vero cell cultures (Fig. 3), as well as in neo-Vero cells exposed to Z-VAD-FMK (not shown). RNA accumulation in cell pellets and shed in supernatants was parallel to that of viral infectivity (not shown), and did not significantly differ in $b c l$-2- and neo-Vero cell cultures.

Taken together, our results show that, in SARS-CoV-infected Vero cells, Bcl-2 expression delayed CPE appearance, abrogated apoptosis, and partially prevented cleavage of both PARP and procaspase 8 . These results indicate that in SARS$\mathrm{CoV}$-infected Vero cells, apoptosis is mediated mainly by the caspase pathway. 
a

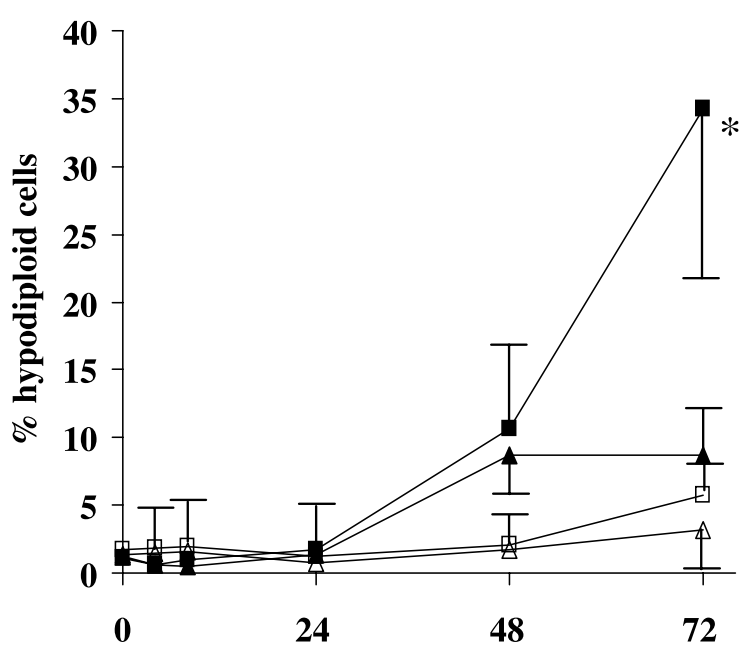

b
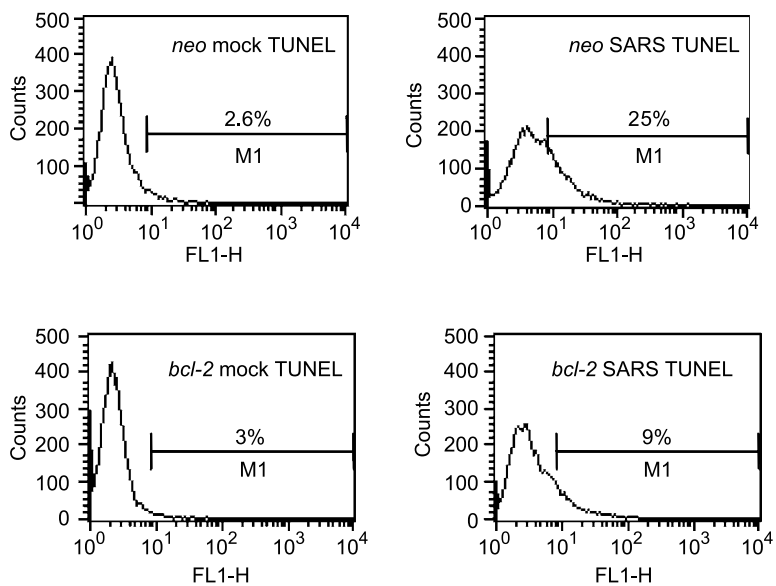

Time from infection (hours)

c
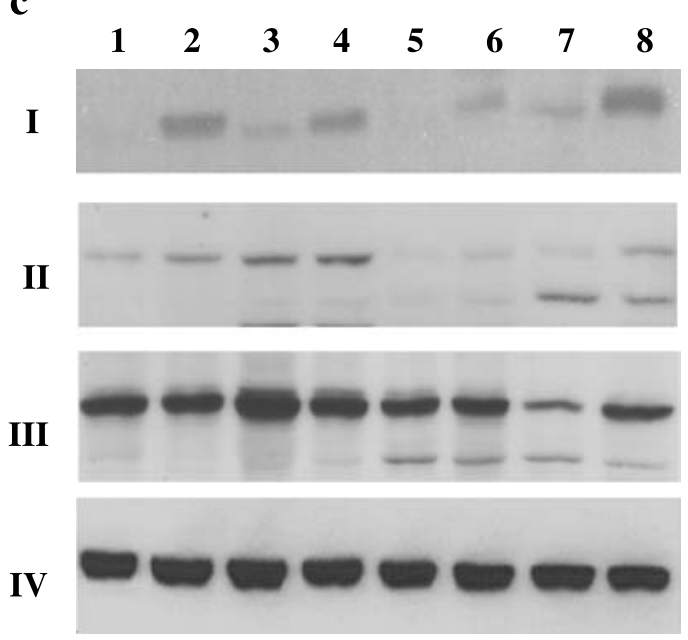

Full length

Cleaved

Full length

Cleaved

Fig. 2. Apoptosis induction by SARS-CoV. a) Hypodiploid cells (as determined by propidium iodide staining) during SARS-CoV infection: $(-\boldsymbol{-})$ neo-Vero cells; $(-\boldsymbol{\Delta}) b c l$-2-Vero cells infected with SARS-CoV; $(-\square)$ mock-infected neo-Verocells; $\left(-\triangle^{-}\right.$mock-infected bcl-2Vero cells. Results are espressed as the \% hypodiploid cells (mean \pm SD out of 4 independent experiments). ${ }^{*} \mathrm{p}=0.008$ b) FACScan analysis of TUNEL. Results of a representative experiment are shown (48 h.p.i.). c) Western Blot analysis of Bcl-2, PARP and procaspase-8 cleavage in $n e o$ - and $b c l$-2-Vero cells. I) Bcl-2; II) PARP; III) procaspase-8; IV) tubulin. Lanes: ( 1 and 2) mock-infected neo- and $b c l$-2-Vero cells, respectively; (3-8) SARS-CoV-infected cells, i.e.: (3) neo-Vero cells, 24 h.p.i.; (4) bcl-2-Vero cells 24 h.p.i; (5) neo-Vero cells, 48 h.p.i.;

(6) bcl-2-Vero cells 48 h.p.i; (7) neo-Vero cells, 72 h.p.i.; (8) bcl-2-Vero cells 72 h.p.i. 

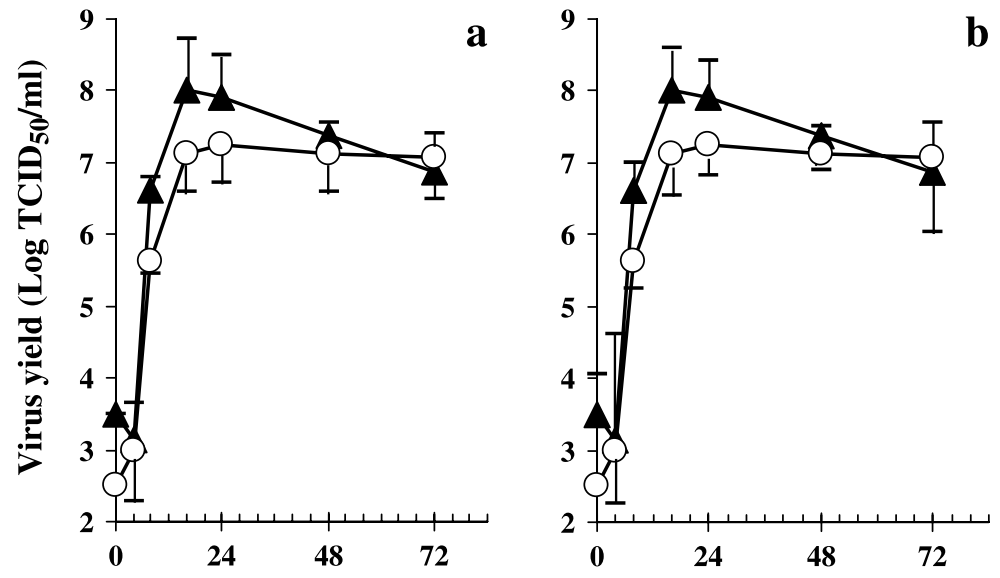

Time from infection (hours)

Fig. 3. Time course of either released $(-\circ-)$ or cell-associated $(\boldsymbol{\Delta})$ infectious virus yield: a) neo-Vero cells, b) $b c l$-2-Vero cells. Results are expressed as a mean $\pm \mathrm{SD}$ from 6 independent experiments

However, apoptosis appears to be a late phenomenon compared with completion of the virus replication cycle. This finding, together with the fact that the inhibition of apoptosis does not alter both kinetics, extent and release of virus progeny from cells, would imply that apoptosis is not involved in facilitating viral release and/or dissemination in SARS-CoV-infected Vero cells.

Although it is not possible to establish whether SARS-CoV-driven apoptosis is a direct consequence of the virus replication, or is mediated by indirect mechanisms, such as the release of pro-apoptotic cytokines, our data can have pathogenetic relevance, since it is likely that the induction of apoptosis is one of the mechanisms involved in the diseased tissue alterations in SARS patients.

\section{Acknowledgments}

Grant support from: European Community "APOCLEAR"; Ricerca corrente e finalizzata del "Ministero della Salute"; COFIN and FIRB-2001 from MIUR. We gratefully acknowledge Dr. Douglas Horejsh for critically reading the manuscript.

\section{References}

1. An S, Chen CJ, Yu X, Leibowitz JL, Makino S (1999) Induction of apoptosis in murine coronavirus-infected cultured cells and demonstration of E protein as apoptosis inducer. J Virol 73: 7853-7859

2. Belyavskyi M, Belavskaya E, Levy GA, Leibowitz JL (1998) Coronavirus MHV-3induced apoptosis in macrophages. Virology 250: 41-49

3. Chan PK, Ip M, Ng KC, Rickjason CW, Wu A, Lee N, Rainer TH, Joynt GM, Sung JJ, Tam JS (2003) Severe acute respiratory syndrome-associated coronavirus infection. Emerg Infect Dis 11: 1453-1454 
4. Chen CJ, Makino S (2002) Murine coronavirus-induced apoptosis in 17Cl-1 cells involves a mitochondria-mediated pathway and its downstream caspase- 8 activation and bid cleavage. Virology 302: 321-332

5. Clem RJ, Feicheimer M, Miller LK (1991) Prevention of apoptosis by a baculovirus gene during infection of insect cells. Science 254: 1388-1390

6. Collins AR (2002) In vitro detection of apoptosis in monocytes/macrophages infected with human coronavirus. Clin Diagn Lab Immunol 9: 1392-1395

7. Reed LJ, Muench H (1938) A simple method of estimating fifty per cent endpoints. Am J Hyg 27: 493-497

8. Dianzani U, Bensi T, Savarino A, Sametti S, Indelicato M, Mesturini R, Chiocchetti A (2003) Role of FAS in HIV infection. Curr HIV Res 4: 405-417

9. Elouet JF, Chilmonczyk S, Besnadeau L, Laude H (1998) Transmissible gastroenteritis coronavirus induces programmed cell death in infected cells through a caspase-dependent pathway. J Virol 72: 4918-4924

10. Haagmans BL, Egberink HF, Horzinek MC (1996) Apoptosis and T-cell depletion during feline infectious peritonitis. J Virol 70: 8977-8983

11. Hay S, Kannourakis G (2002) A time to kill: viral manipulation of the cell death program. J Gen Virol 83: 1547-1564

12. Lee SK, Youn HY, Hasegawa A, Nakayama H, Goto N (1994) Apoptotic changes in the tymus of mice infected with mouse hepatitis virus, MHV-2. J Vet Sci 56: 879-882

13. Levine B, Huang O, Isaacs JT, Reed JC, Griffin DE, Hardwick JM (1993) Conversion of lytic to persistent alphavirus infection by the $b c l-2$ cellular oncogene. Nature 361: 739-742

14. Li ML, Stollar V (2004) Alphaviruses and apoptosis. Int Rev Immunol 1-2: 7-24

15. Liu C, Xu HY, Liu DX (2001) Induction of caspase-dependent apoptosis in cultured cells by the avian coronavirus infectious bronchitis virus. J Virol 75: 6402-6409

16. Mazzulli T, Farcas GA, Poutanen SM, Willey BM, Low DE, Butany J, Asa SL, Kain KC (2004) Severe acute respiratory syndrome-associated coronavirus in lung tissue. Emerg Infect Dis 10: 20-24

17. Ng ML, Tan SH, See EE, Ooi EE, Ling AE (2003) Proliferative growth of SARS coronavirus in Vero E6 cells. J Gen Virol 84: 3291-3303

18. Nicoletti I, Migliorati G, Pagliacci MC, Grignani F, Riccardi C (1991) A rapid and simple method for measuring thymocyte apoptosis by propidium iodide staining and flow cytometry. J Immunol Methods 139: 271-279

19. Paltrinieri S, Ponti W, Comazzi S, Giordano A, Poli G (2003) Shifts in circulating lymphocyte subset in cats with feline infectious peritonitis (FIP): pathogenic role and diagnostic relevance. Vet Immunol Immunopathol 96: 141-148

20. Peiris JSM, Chu CM, Cheng VC, Chan KS, Hung IF, Poon LL, Law KI, Tang BS, Hon TY, Chan CS, Chan KH, Ng JS, Zheng BJ, Ng WL, Lai RW, Guan Y, Yuen KY; HKU/UCH SARS Study Group (2003) Clinical progression and viral load in a community outbreak of coronavirus-associated SARS pneumonia: a prospective study. Lancet 361: 1767-1772

21. Quinfen Z, Jinming C, Xiaojun H, Huanying Z, Jicheng H, Ling F, Kunpeng L, Jingqiang Z (2004) The life cycle of SARS coronavirus in Vero E6 cells. J Med Virol 73: 332-337

22. Scallan MF, Allsopp TE, Fazakerley JK (1997) bcl-2 acts early to restrict semliki forest virus replication and delays virus-induced programmed cell death. J Virol 71: 1583-1590

23. Sungwhan A, Chen C, Yu X, Leibowitz JL, Makino S (1999) Induction of apoptosis in Murine coronavirus-infected cultured cells and demonstraction of $\mathrm{E}$ protein as a apoptosis inducer. J Virol 73: 7853-7859 
24. Tan YJ, Fielding BC, Goh P-Y, Shen S, Tan THP, Lim SG, Hong W (2004) Overexpression of $7 \mathrm{a}$, a protein encoded by the severe acute respiratory syndrome coronavirus, induces apoptosis via a caspase-dependent pathway. J Virol 78: 14043-14047

25. Teodoro JG, Branton PE (1997) Regulation of apoptosis by viral gene products. J Virol 71: $1739-1746$

26. Tewari M, Dixit VM (1995) Fas-and tumor necrosis factor-induced apoptosis is inhibited by the poxvirus crmA gene product. J Biol Chem 270: 3255-3260

27. Yan H, Xiao G, Zhang J, Hu Y, Yuan F, Cole DK, Zheng C, Gao GF (2004) SARS coronavirus induces apoptosis in Vero E6 cells. J Med Virol 73: 323-331

Author's address: Dr. Maria R. Capobianchi, Laboratory of Virology, National Institute for Infectious Diseases (INMI), “L. Spallanzani”, Via Portuense, 292, 00149 Rome, Italy; e-mail: capobianchi@inmi.it 